\title{
ESTRATEGIAS DE RESISTENCIA, REPRODUCCIÓN SOCIAL Y SUPERACIÓN DEL CAMPESINADO DE MARINALEDA FRENTE A LA CRISIS DEL CAPITALISMO NEOLIBERAL (PROVINCIA DE SEVILLA, ESPAÑA $)^{1}$
}

\author{
STRATEGIES FOR RESISTANCE, SOCIAL REPRODUCTION \\ AND OVERCOMING THE MARINALEDA CAMPESINATO \\ AGAINST THE CRISIS OF NEOLIBERAL CAPITALISM \\ (PROVINCE OF SEVILLE, SPAIN)
}

\author{
Dorival Borelli Filho \\ Universidade Estadual Paulista, Instituto de Geografia, Rio Claro, SP, Brasil \\ borellifilho@yahoo.com.br \\ José Gilberto de Souza \\ Universidade Estadual Paulista, Instituto de Geografia, Rio Claro, SP, Brasil \\ jg.souza@unesp.br
}

\begin{abstract}
Resumen
El presente estudio objetiva informar las estrategias de resistencia, reproducción y superación del campesinado territorializado en la comunidad agrícola de Marinaleda (Provincia de Sevilla, España) frente a la crisis del capitalismo neoliberal de 2008. En este sentido, se pretende en esta investigación analizar en la referida espacialidad los procesos de ocupación liderados por movimientos sociales campesinos, la implementación de sistemas cooperativos y adopción de estrategias de producción/comercialización colectiva, así como, estrategias establecidas con el poder público local y procesos de consolidación de políticas públicas con efectiva participación de la comunidad, como forma de determinación territorial campesina.
\end{abstract}

Palabras-clave: Estrategias de resistencia. Reproducción social. Superación. Campesinado. Crisis del capitalismo neoliberal.

\begin{abstract}
The present objective study to report the strategies of resistance, reproduction and overcoming of the territorialized peasantry in the agricultural community of Marinaleda (Province of Seville, Spain) in the face of the neoliberal capitalism crisis of 2008. In this sense, it is intended in this research to analyze in the referred spatiality the occupation processes led by peasant social movements, the implementation of cooperative systems and adoption of collective production/commercialization strategies, as well as, strategies
\end{abstract}

\footnotetext{
${ }^{1}$ Estágio de Pesquisa no Exterior realizado na Universidade de Córdoba (UCO) - Espanha, entre 01/05/2014 a 31/07/2014, mediante concessão de Bolsa BEPE pela Fundação de Amparo à Pesquisa do Estado de São Paulo. Processo FAPESP: 2013/25870-8.
} 
Estrategias de resistencia, reproducción social y superación

Dorival Borelli Filho

del campesinado de marinaleda frente a la crisis del

José Gilberto de Souza

capitalismo neoliberal (Provincia de Sevilla, España)

established with the local public power and processes of consolidation of public policies with effective participation of the community, as a form of peasant territorial determination.

Keywords: Resistance strategies. Social reproduction. Autonomy. Peasantry. Neoliberal capitalism.

\section{Introducción}

Precipitada por la falencia del tradicional banco de inversiones estadunidense Lehman Brothers y de la seguradora American International Group (AIG), la Crisis Financiera de 2008 puede ser interpretada por distintos ángulos: como una crisis de las finanzas globales desreguladas o como una crisis de la ausencia de reglamentación del aparato estatal sobre el mercado financiero, originada en la "burbuja de la internet" (2001), o en un segmento específico del sistema financiero norte-americano y propagada para otros sectores de la economía mundial a partir de hipotecas de mercado subprime, donde había se creado una pirámide artificial de títulos securitarios sofisticados que desmoronaron.

Iniciada en el sistema inmobiliario norte-americano, en pocas semanas, la crisis se cambió en un movimiento sistémico que atingió el continente europeo, impactando, fuertemente, el territorio español. Datos oficiales del Instituto Nacional de Estadística (INE) referentes al primero trimestre de 2013, demostraron que el desempleo continuó en un movimiento ascendente en España, alcanzando 27,16\% entre la Población Económicamente Activa (PEA), registrando la "cuarta economía" de la Zona del Euro 6.202.700 millones de desempleados (SEGRE, 2013).

En este contexto de crisis del capitalismo neoliberal, el discurso político propalado por el poder público de Marinaleda, afirmó que el pueblo se sobresale como una localidad que no fue profundamente atingida por la crisis del mercado financiero mundial, manteniéndose inmune y caracterizándose como una isla de prosperidad y tranquilidad, viviendo una situación de pleno empleo, situando el desempleo entre $0 \%$ a 4\% de la población económicamente activa, con una renta media de 47 euros por día (1.128 euros al mes).

Adoptamos en este estudio para el análisis de la producción del territorio campesino en la comunidad agrícola de Marinaleda (Provincia de Sevilla, España) la 
Estrategias de resistencia, reproducción social y superación

Dorival Borelli Filho

del campesinado de marinaleda frente a la crisis del

José Gilberto de Souza

capitalismo neoliberal (Provincia de Sevilla, España)

concepción de territorio que ha sido desarrollado por Souza (2009) como un conjunto de relaciones sociales fundadas en relaciones de poder. En este sentido, el territorio es la primera manera de dar sentido a las relaciones de poder y los cambios en la organización de las relaciones sociales corresponden a los cambios en las representaciones del poder.

La esta investigación se le concedió un carácter empírico, mediante la realización de trabajos de campo que se basa en una recopilación de datos a través de entrevistas con los residentes de la comunidad de agricultores y líderes políticos locales, tratando de identificar el grado en que los aspectos socio-territoriales asisten como elementos de resistencia y de superación de la crisis del capitalismo financiero. La definición de la muestra específica de los sujetos de investigación a ser entrevistadas se basa en el número de residentes directa y diaria que participan en el sistema de cooperación (42 cooperativas), con la intención de alcanzar el porcentaje del 10\% de los encuestados.

La investigación de campo en Marinaleda ocurrió entre los meses de mayo y julio de 2014 y, en este sentido, los datos presentados en este estudio se refieren a un momento socio-político-económico-histórico particular experimentada por la Zona Euro, los campesinos y los trabajadores de cooperación espacial y territorializada de la región de la comunidad andaluza.

\section{Los elementos de resistencia frente a la crisis del capitalismo neoliberal}

En Marinaleda, el modelo que se encuentra establecido para superar la crisis del capitalismo neoliberal, congrega un proceso de reforma agraria iniciada en los años 80 , la creación de una cooperativa agrícola, un programa de vivienda popular, de bajo costo de vida en las zonas rurales y la estabilidad política proporcionada por un gobierno de carácter socialista, que sostiene sucesivamente en el poder en la ciudad desde hace más de tres décadas y eso no importa para medir el desarrollo del pueblo en términos cuantitativos o económicos, pero la calidad de vida y/o el bienestar de su población.

A pesar de la Cooperativa Humar - Marinaleda SCA no emplear toda la población de la ciudad, alrededor de 2700, la granja y la forma cooperativa constituyen la base económica del lugarejo, estimular y sosteniendo todo el pueblo, causando baja tasa de desempleo. Las autoridades locales afirman que alrededor del $80 \%$ de la población de la aldea estaría involucrado directa o indirectamente con las actividades de la cooperativa. El 
Estrategias de resistencia, reproducción social y superación

Dorival Borelli Filho

del campesinado de marinaleda frente a la crisis del

José Gilberto de Souza

capitalismo neoliberal (Provincia de Sevilla, España)

resto de la población de Marinaleda se emplea en el sector de servicios, especialmente, en las pequeñas empresas familiares y en la "construcción civil", sobre todo, en el programa de viviendas de populares instituidas por el gobierno local.

Con su cooperativa agrícola, según los datos lanzados por el gobierno local, el pueblo llega a una situación de pleno empleo, la tasa de desempleo oscila entre el $0 \%$ y $4 \%$, y aunque ningún aldeano del pueblo tiene deuda hipotecaria, debido al programa de vivienda asequible establecida por el gobierno municipal.

Después de que el proceso de expropiación de la Finca de "El Humoso", en años 80 , los trabajadores rurales se dan cuenta de que sólo la conquista de la tierra no garantizaría su reproducción social. En el curso de la década de 90, cuando se completa el proceso de implementación del sistema de riego en toda la propiedad, se introdujo un sistema de producción cooperativa, estableciéndose una fábrica de conservas que garantiza el fornecimiento de productos de valor añadido para las cooperativas (alcachofas, pimientos, habas y aceite de oliva), aun destacando la crianza de ovejas para corte, con un ejemplo concreto de cooperativización de la tierra, de producción agrícola, de procesamiento agroindustrial.

En este sentido, hay que añadir que el pueblo no es el medio de producción capitalista (terratenientes contra los jornaleros) que determinan las relaciones de producción de los trabajadores agrícolas, pero éstos, por la colectivización de la tierra y de la agroindustrialización, decidir colectivamente cómo y cuánto trabajar, controlando los medios de producción en todas sus fases, desde la producción de plántulas en invernaderos hasta la comercialización de conservas de la cooperativa.

Parece que la parte superior de su producción, la Cooperativa Humar cuenta con 500 miembros, que reciben $€ 47$ por día, totalizando en promedio y un "techo" de 1.200 euros al mes, independiente de los trabajos que realicen (de campo, logística, producción, el comercio y la línea administrativa), mientras que el salario mínimo nacional se mantiene congelado en $€ 645$ en el gobierno español.

En términos de la seguridad social, los días no laborables son "cubiertos" por la cooperativa. En los cultivos que no requieren una gran cantidad de mano de obra (alcachofa), el régimen de trabajo de la cooperativa consiste en seis días a la semana, por un total de 36 horas por semana, en un turno que se extiende de $6 \mathrm{~h}$ a $14 \mathrm{~h}$, empleando al menos 40 trabajadores, pero puede llegar a 80 a 90 personas en la línea de 
Estrategias de resistencia, reproducción social y superación

Dorival Borelli Filho

del campesinado de marinaleda frente a la crisis del

José Gilberto de Souza

capitalismo neoliberal (Provincia de Sevilla, España)

producción, cuando el período de industrialización de pimientos del piquillo, formando dos grupos de trabajo.

La producción agrícola en "El Humoso" ocurre durante todo el año, ocupando el terreno disponibles, por exceso de las zonas de barbecho, con el uso de implementos agrícolas y sistemas de riego, con el uso de la tecnología tanto en el campo, como en agroindustria, que, a su vez, revelan la constante búsqueda de los campesinos para adquirir los medios de producción indispensables para la producción agrícola.

La cantidad de la mano de obra empleada en el campo y en la Cooperativa Humar depende del tipo de cultivo que se celebrará en "El Humoso", que se caracteriza, en ciertas épocas del año, como la temporada, ya que, por ejemplo, el cultivo y la cosecha de alcachofa no requiere una gran cantidad de trabajadores, sólo se requiere un turno de labor. En este sentido, debe considerarse que el cultivo de pimientos recolectados en agosto, requiere un mayor número de trabajadores, el período en que se producen la contratación de más trabajadores, así como el haba, que requiere 400 trabajadores, recolectadas al medio del período de la alcachofa y debe ser procesada rápidamente, sin ningún día de descanso laboral.

En este sentido debe aclararse que, el cultivo de pimientos (piquillo) y haba son entendidas por el gobierno municipal como "agricultura social", es decir, que no se producen con el fin de contribuir ganancias económicas significativas a la cooperativa, pero para la generación de empleo en el pueblo. Sin embargo, en momentos en que el trabajo escasea en "El Humoso," este es compartido, repartido entre los trabajadores rurales, que se produce una rotación semanal de los grupos de trabajo, a fin de conservar los puestos de trabajo y no generan el desempleo en la comunidad.

En función del tipo de cultivo y la mano de obra requerida, los trabajadores pueden moverse entre el campo y la inversa cooperativa y al revés, y también pueden trabajar en las ocho asociaciones cooperativas administradas por el Sindicato de Obreros del Campo (SOC) no trabajan en separado, sobre todo, a causa de la colectivización de la tierra. En este sentido, se considera que los puestos de trabajo no son considerados por los trabajadores como una propiedad en concreto, siguiendo tales empleados la ideología de "trabajar menos para que todos trabajen". En tiempos malos para la agricultura, debido a las condiciones climáticas, los agricultores de "El Humoso" 
Estrategias de resistencia, reproducción social y superación

Dorival Borelli Filho

del campesinado de marinaleda frente a la crisis del

José Gilberto de Souza

capitalismo neoliberal (Provincia de Sevilla, España)

trabajan al menos siete meses al año, pero puede llegar a 11 meses en los cultivos productivos.

La Cooperativa Humar también ha adquirido y procesado la producción de pequeños granjeros campesinos, pero sin ningún tipo de contrato o sociedad, contribuyendo, de esta manera, con su reproducción social. Al final del año agrícola, después de liquidados todos los costos de producción, los beneficios adquiridos como la producción agrícola y la agroindustrialización no se distribuyen entre los trabajadores de las cooperativas, pero invertidos en nuevos cultivos y en la propia cooperativa, sobre todo, en la adquisición de nuevos maquinarios y aperos agrícolas, con el fin de emplear a un mayor número de personas en la próxima campaña agrícola.

En cuanto a los flujos de producción, los enlatados producidos por la Cooperativa Humar son, especialmente, comercializados en las Regiones de las Comunidades Autónomas de Andalucía, Navarra, País Vasco y con Italia, aún siendo que, el aceite de oliva se comercializa directamente (con el logo de la cooperativa) entre el Gobierno de Marinaleda con el Gobierno de Venezuela, no teniendo como intermediario el gobierno español.

Al principio, una de las estrategias utilizadas por los gestores de la cooperativa, con el fin de evitar la acción de un intermediario, y por lo tanto, un proceso de extracción de renta de la tierra por el capital comercial es la comercialización de los productos industriales directamente al consumidor final en la sede de propia cooperativa, a través la página web de la cooperativa (individual o por lotes), y en el comercio local, que no puede absorber toda la producción de la cooperativa, pero asegura que la obtención de una renta de monopolio.

La estrategia de comercialización (internet y tienda), aunque sumadas, corresponde a un pequeño porcentaje de las ventas de la cooperativa, que van del 15\% al $20 \%$, que son adquiridos por las asociaciones o por pequeños consumidores, que están pasando a través de la ciudad y quieren consumir o comprar uno de los principales símbolos de Marinaleda.

Los productos agrícolas producidos en "El Humoso" y procesados en la cooperativa son también comercializados con el capital comercial e incluso sin el logo de la cooperativa, denominado por los administradores como "marca blanca", lo que representa alrededor del $80 \%$ de las ventas de aceite de oliva, hecho que se justifica por 
Estrategias de resistencia, reproducción social y superación

Dorival Borelli Filho

del campesinado de marinaleda frente a la crisis del

José Gilberto de Souza

capitalismo neoliberal (Provincia de Sevilla, España)

el desconocimiento de su marca en el mercado, pero que muestra una extracción o una subordinación de los ingresos de los campesinos, por los bajos precios pagados por los productos agrícolas y una pérdida identidad de la Cooperativa Humar, considerado uno de los símbolos de Marinaleda, cuyo desarrollo se debe en parte a raíz del capitalismo comercial.

Por estar subordinadamente inserida en el mercado, a través de la venta de sus productos agrícolas a través de la Cooperativa Humar, el pueblo está en parte afectado por la crisis, principalmente debido a los bajos precios pagados por las cadenas de supermercados de sus conservas, pero eso no ha sacudido profundamente su economía. También la villa es atingido vía institucionalmente, mediante la reducción de las transferencias corrientes a las arcas municipales y el aumento de la demanda de empleo en el campo, es decir, el retorno de los residentes que antes de la crisis eran empleados en el negocio de la construcción civil en otros municipios, especialmente, en Málaga, situada a unos $100 \mathrm{~km}$ de Marinaleda.

Ante esta situación de búsqueda de empleo en el campo, el gobierno ha tratado de idear nuevas alternativas, con el fin de generar nuevos puestos de trabajo, con la llamada "agricultura social", intensificando la agroindustrialización y dando ligereza a la transición agroecológica, cuyas ganancias económicas son más significativas.

Además de retener una parte de la renta de la tierra, por los bajos precios pagados por los productos agrícolas, el capital comercial también ha establecido los tipos de cultivo en "El Humoso", especialmente, en los "cultivos sociales" y, por tanto, interfiere en la generación puestos de trabajo en la ciudad.

A pesar de la Cooperativa Humar no haber realizado en la Finca de "El Humoso" una transición agroecológica completa después de 23 años de despojo de la propiedad, de esta manera, se encuentra en contradicción con los principios de la reforma agraria, la producción de alimentos saludables, tal contradicción no puede ser entendida como una descalificación de un proceso histórico de lucha por la tierra y resistencia a un modelo de sociedad de consumo, sobresaliendo el hecho de que la tierra conquista, aparece como un territorio delimitado, rodeado por la propiedad privada con cultivos con uso intensivo de agroquímicos.

La transición de la Finca "El Humoso" para una matriz agroecológica se está produciendo poco a poco, de acuerdo con el interés del mercado, el flujo de producción, 
Estrategias de resistencia, reproducción social y superación

Dorival Borelli Filho

del campesinado de marinaleda frente a la crisis del

José Gilberto de Souza

capitalismo neoliberal (Provincia de Sevilla, España)

situadas en una fase llamada Agricultura de la Producción Integrada (API), con el uso de los productos controlados (agro tóxicos) aplicados en el campo, lo que, en su opinión, es un paso previo a la producción agroecológica.

Reconociendo la contradicción entre el modelo convencional de agricultura desarrollado una parte importante de uno de la Finca "El Humoso" y uno de los paradigmas de la reforma agraria, la producción de alimentos sanos, la intención del gobierno es la transición completa a la matriz agroecológica, pero sin un plazo previsto, porque en un momento de crisis de los mercados, las atenciones se centran en la generación de más puestos de trabajo, a través de la "agricultura social" y la producción agroecológica empieza a suponer un paso siguiente.

En relación al centro industrial de la ciudad, se debe aclarar que la Cooperativa Humar es la única instalación existente en el lugar, lo que indica la falta de interés por parte del gobierno municipal en atraer otras empresas y/o inversiones a la ubicación, que a su vez, puede provocar la pérdida de control por parte de Juan Manuel Sánchez Gordillo (Alcalde de Marinaleda) del territorio conquistado y, en consecuencia, sobre las relaciones sociales, de poder y producción, instituidos desde la década de 1980.

El programa de viviendas populares idealizado por el gobierno en el pueblo llamado "viviendas de autoconstrucción" o "Casas de la Revolución", básicamente consiste en la adquisición de tierras por el ayuntamiento de terrenos considerados "rústicos" a precios ínfimos, que son luego urbanizados y así valorizados. Los materiales de construcción son subsidiados por el Estado, a través de transferencia de recursos de la Junta de Andalucía. Sin embargo, las medidas drásticas, con la negativa del propietario de vender la tierra, esta propiedad puede ser expropiada incluso por el Gobierno Municipal de Marinaleda.

Tal programa de vivienda populares posee como objetivo envolver el futuro residente durante todo el proceso de construcción de viviendas, construidas colectivamente y de forma simultánea, es decir, tiene la obligación de trabajar en sus casas, sin saber, sin embargo, cual va a ser su "propiedad", porque al final de las construcciones es realizado un sorteo, en asamblea pública, con el fin de definir cuál residente ocupará cierta vivienda. Existe también la posibilidad en el programa de la contratación de mano de obra pagada por el beneficiario, además de pagar por el 
Estrategias de resistencia, reproducción social y superación

Dorival Borelli Filho

del campesinado de marinaleda frente a la crisis del

José Gilberto de Souza

capitalismo neoliberal (Provincia de Sevilla, España)

servicio albañiles profesionales, con el fin de realizaren actividades más complejas, generando, de esta manera, empleo en el pueblo.

De los relatos recolectados a través de trabajo de campo, las autoridades locales afirman que, en este sistema de trabajo implementado por el programa de vivienda, se necesita alrededor de 400 días de trabajo para la realización de las obras, no necesariamente consecutivos, y todos los auto-constructores, acompañados por un arquitecto pagado por el gobierno, debe tener el mismo número de días de trabajo.

Esas viviendas se amortizan a 15 euros por mes (con el fin de indemnización por daños materiales construcción subvencionada por la Junta de Andalucía), que se transforman en una especie de alquiler social, en el que los beneficios son propensos a ser reembolsados por sus hijos o nietos, que obtendrán al final del proceso la posesión de la propiedad.

A partir de las condiciones establecidas, el gobierno de la ciudad quiere evitar un proceso de especulación inmobiliaria en la municipalidad, a través de los sistemas de construcción y sistemas bancarios, ya que el valor total de las casas, construidas en un modelo estándar, incluyendo en su pintura, con superficie construida de $90 \mathrm{~m}^{2}$ y $100 \mathrm{~m}^{2}$ de patio para acomodar expansiones futuras, llega a un costo total de 20 mil euros.

Incluso al final del pago de la propiedad, el autoconstructor se convierte en propietario, pero, al parecer, no consiguen un título de propiedad de la casa, y no puede, por lo tanto, ser comercializada, excepto con el gobierno municipal, que paga por la vivienda sólo la cantidad de días trabajados por autoconstructor y no reembolsan el valor de los beneficios quitados, que las revenden al mismo valor, no especulando, de esta manera, con el inmóvil.

En este programa, existen flexibilidades, pues estas viviendas pueden ser alquiladas, pero el valor de los beneficios (15 euros). Hasta el inicio de este programa de vivienda asequible, que benefició a 350 familias. Los criterios adoptados para la selección de auto-constructores son: matrimonio con hijos, parejas sin hijos y persona soltera, siendo que, en las construcciones recientes solteros ya están beneficiándose. Caso la propiedad no está ocupado por el arrendatario, el gobierno puede expropiar y revenderlo.

Parcialmente separada del gran capital y caracterizada por una cierta nivelación social y un pequeño comercio de carácter familiar, en Marinaleda, el sistema capitalista 
Estrategias de resistencia, reproducción social y superación

Dorival Borelli Filho

del campesinado de marinaleda frente a la crisis del

José Gilberto de Souza

capitalismo neoliberal (Provincia de Sevilla, España)

aún no se estableció, con sus grandes cadenas de supermercados, sistema bancario, electrónicos, cadenas de comida rápida, etc., no observándose de esta manera, esta espacialidad, el materialismo y el consumismo exacerbado vistos en los grandes centros urbanos españoles.

En los institutos de educación, los libros se adquieren colectivamente, lo que los hacen más baratos, y trasladados a los estudiantes de los ciclos siguientes, siendo también la cafetería de los colegios accesibles a los estudiantes, con cargo de 13 euros al mes, así como la guardería que cuesta 2 euros por mes y el gimnasio, que tiene un costo mensual de 2 euros, así como una piscina pagada 3 euros/mes, que revelan el bajo costo de vida en el pueblo.

En razón de las dimensiones de las viviendas, que tienen patios, existe la costumbre entre los residentes de producir alimentos agrícolas en la parte posterior de sus hogares, así como la crianza de animales pequeños, complementando parte de su alimentación, en un tipo de producción agrícola y de autoconsumo y que pueden ser consideradas como una especie de habitus campesina establecida en el espacio urbano.

\section{Consideraciones finales}

A pesar de Marinaleda haber sido constituida una singularidad, a no ser por lo menos tan profundamente impactada por la crisis capitalista neoliberal de 2008 , lo que generó una serie de reportajes acerca de este lugar, el modelo de desarrollo social, económico y político implementado en el pueblo por el Colectivo Unitario de los Trabajadores, que se ha desarrollado desde mediados de la década de 1980, se mantuvo restringida a esta espacialidad, no se expandiendo por otras ciudades andaluzas, a pesar de numerosos procesos de ocupación engendradas por el SOC.

Al que todo indica, el Alcalde Juan Manuel Sánchez Gordillo ha establecido en esta espacialidad, un centralismo político, coadunado con consultas populares, interfiriendo e/ influyendo en todas las relaciones políticas y productivas del pueblo, pasando por el ejercicio del poder ejecutivo municipal, por el parlamentar, el control sobre el Consejo Municipal y que todavía alcanza el SOC y la Cooperativa Humar.

Marinaleda, con su lucha por la tierra, con la aplicación de su sistema cooperativo, que todavía cubren los programas de vivienda popular, el compartimento del trabajo agrícola llamado "cultivos sociales", genera no sólo un modelo alternativo a 
Estrategias de resistencia, reproducción social y superación

Dorival Borelli Filho del campesinado de marinaleda frente a la crisis del José Gilberto de Souza capitalismo neoliberal (Provincia de Sevilla, España)

los paradigmas establecidos de la administración pública, convirtiéndose no sólo, como una utopía para la paz, sino como una alternativa a los mercados, como el intento de crear una realidad social más justa e igualitaria, donde las relaciones sociales no son guiados por el predominio de la propiedad privada de los medios de producción, especialmente, de la tierra y del espacio urbano, sino por el espíritu colectivo y cooperativo de su población, pueblo andaluz donde las palabras solidaridad y la singularidad son clave.

El pueblo consiguió adoptar otros valores, lo que demuestra que un modelo alternativo de sociedad es posible y el desarrollo de un dispositivo político, económico y social único, que hizo detener el desempleo sistémico, cesando la inmigración y atrayendo a los residentes más antiguos. El compromiso de los residentes de la localidad andaluza bajo el liderazgo de un sindicalista posibilitó poner una experiencia política y económica en curso real, en una especie de isla comunista de resistencia en el centro de los campos capitalistas andaluces y en la sociedad de consumo urbano español, superando las sucesivas crisis del mercado neoliberal.

\section{REFERÊNCIAS}

SEGRE, M. Profundizarse a la crisis en España y la solución favorece el capital. 30/05/2013.

SOUZA, J. G. Limites de território. Agraria, São Paulo, n. 10/11, pp. 99-30, 2009.

Recebido em 16/09/2019.

Aceito para publicação em 03/06/2020. 